\title{
LA REFORMA DE LAS ENSEÑANZAS UNIVERSITARIAS DESDE EL ANGULO INTERNACIONAL
}

\author{
RICARDO MARIN IBAÑEZ \\ Universidad Nacional de Educación a Distancia
}

Por imperativo legal, en desarrollo de la Ley Orgánica de Reforma Universitaria de 1983 y a la vez de los estatutos de las respectivas universidades, van a ser renovados los planes de estudios.

El tema, pues, merece una consideración especial. Nosotros vamos a enfocarlo desde el triple ángulo: los artículos de la Ley que conciernen a nuestro tema; las propuestas formuladas por el Consejo de Universidades; y la reflexión crítica sobre las directrices elaboradas por el Consejo a la luz de la información internacional.

\section{BASES LEGALES}

La Ley de Reforma Universitaria de 1983 impone un nuevo marco de referencia, al que tendrá que adaptarse la actuación de las Universidades en el ejercicio de su autonomía.

Recordemos los artículos decisivos para el tema del curriculum:

"28.1 El Gobierno, a propuesta del consejo de universidades, establecerá los títulos que tengan carácter oficial y validez en todo el territorio nacional, así como las directrices generales de los planes de estudio que deban cursarse para su obtención y homologación."

"28.3 Las Universidades, en uso de su autonomía, podrán inpartir enseñanzas conducentes a la obtención de otros diplomas y títulos."

"29.1 Con sujeción a lo dispuesto en el artículo anterior, las Universidades elaborarán y aprobarán sus planes de estudio, en los que se señalarán las materias que para obtención de cada título deben ser cursadas obligatoria y optativamente, los periodos de escolaridad y los trabajos o prácticas que deben realizar los estudiantes."

"30. Los estudios universitarios se efectuarán, como máximo, en tres ciclos. La superación del primero de ellos dará derecho, en su caso, a la obtención del título de Diplomado, de Arquitecto Técnico o de Ingeniero Téc- 
nico; la del segundo, a la del título de Licenciado, de Arquitecto o de Ingeniero, y la del tercero a la del título de Doctor. En su caso se establecerán las condiciones de convalidación o adaptación para el paso de un ciclo a otro".

Para cerrar estas citas seleccionamos del prólogo de la Ley lo que tiene más directa vinculación con el tema del curriculum:

"La previsible incorporación de España al área universitaria europea supondrá una mayor movilidad de titulados españoles y extrangeros, y se hace necesario crear el marco institucional que permita responder a este reto a través de la adaptación de los planes de estudio y la flexibilización de los títulos que se ofertan en el mercado de trabajo... De acuerdo con dicho doble objetivo docente e investigador se potencia la estructura departamental de las Universidades españolas, lo que debe permitir no sólo la formación de equipos coherentes de investigación, sino también una nota flexibilizadora de los currícula que pueden ser ofertados".

\section{LOS DOCUMENTOS DEL CONSEJO DE UNIVERSIDADES}

Vamos a utilizar dos textos en donde aparece la doctrina sustentada por el Consejo de Universidades: el "Documento elaborado por la Ponencia de Reforma de Enseñanzas Universitarias" (en adelante designado como "Documento") que fue aprobado por el Pleno del Consejo de Universidades el 30 de Enero de 1986 y "La Reforma de las Enseñanzas Universitarias" (Reforma) publicado en Febrero de 1987 y que ha tenido amplia difusión.

Estos documentos intentan trazar las líneas fundamentales de una Reforma de la Enseñanza Superior que sin duda arranca de la legislación precedente, especialmente de la Ley de Educación de 1970, pero a la vez revela un profundo impacto de la educación anglosajona, explicable no sólo por razones personales - ya que en ese ámbito se formaron gran parte de los que han formulado este programa- sino sobre todo por la influencia sociopolítica y cultural de los Estados Unidos e Iglaterra, que han desplazado al modelo alemán y al francés tradicionalmente determinantes de nuestras enseñanzas universitarias.

Veamos algunos de los puntos decisivos de estos textos

\subsection{Objetivos.}

Los objetivos vienen formulados explícitamente en la "Reforma"

1.. Actualizar las enseñanzas y conocimientos que se imparten en las Universidades españolas incorporando otras nuevas que el desarrollo cultural, científico y técnico exige, facilitando la formación interdisciplinar e in- 
cluyendo en los currícula universitarios enseñanzas instrumentales (como lenguas modernas o informatica) que deben construir hoy parte del bagaje intelectual de todo universitario.

2.. Flexibilizar las enseñanzas que se imparten de modo que, el carácter estatal de los títulos académicos, reconocido en el artículo 149.1.30 de la Constitución española, se armonice con la autonomía de las universidades y ésta con el respeto a los intereses de los estudiantes. Así pues, los planes de estudio conducentes a un mismo título oficial podrán variar de una a otra Universidad y, en una misma Universidad, podrán variar también los currícula de los estudiantes en orden a la obtención de un mismo título oficial, potenciandose así la optatividad de los estudios que cursará cada estudiante.

39. Vincular Universidad y sociedad, aproximando las enseñanzas a las necesidades sociales.A ello responde, aparte la propia flexibilidad de los planes de estudio, la ordenación cíclica de las enseñanzas, que debe permitir una alternancia entre estudio y trabajo, contribuyendo a la disminución del fracaso escolar. Al mismo objetivo se orienta la diversificación del catálogo de títulos oficiales y, sobre todo, de las especializaciones que podrán ofertar libremente las Universidades.

4.. Finalmente, la reforma pretende también adaptar el sistema de enseñanza superior a los requerimientos derivados de diversas directivas de la CEE. De una parte, hay directivas que regulan enseñanzas especificas como son las de medicina, farmacia, veterinaria, arquitectura y otras. De otra, debe tenerse en cuenta que textos importantes en tramitación en la CEE prevén un módulo básico de tres años como preparación inicial para la práctica profesional. Por último, y con carácter genérico, la incorporación de España al área educativa y cultural europea exige homologar y armonizar nuestra ordenación académica (títulos, ciclos y planes) a la de los países más avanzados.

\subsection{Títulos}

Como sugiere la Ley de Reforma Universitaria hay una clara distinción entre "los títulos que tengan carácter oficial y validez dentro del territorio nacional" que tienen que ser establecidos por el Gobierno a propuesta del Consejo de Universidades y "otros diplomas y títulos", que las universidades podrán otorgar en uso de su autonomía.

La designación de "título" se aplicará a las enseñanzas que reclamen para su comienzo los requisitos generales de acceso a la universidad, en tanto que los "diplomas" tienen más bien un carácter de extensión universitaria, o de cursos de especialización, impartidos por institutos y departamentos universitarios. 
Incluso cuando se trate de los títulos referidos en el artículo 30 (diplomado, licenciado, , ingeniero...) unos podrán tener carácter oficial y validez nacional, y otros serán propios de las respectivas universidades.

Un rasgo interesante es el de la estricta viculación que exitía entre titulación y Centro, de manera que a cada Escuela o Facultad le correspondía un sólo título, y cuando se deseaba alcanzar un título diferente había de crear un Centro distinto. Con la nueva situación se contempla la ruptura de esta vinculación. Un Centro puede impartir varios títulos y un título puede ser alcanzado en planes de estudios conjuntos, impártidos en dos o más universidades españolas y extranjeras, supuestos los convenios correspondientes.

Esta situación se da en muchas universidades, especialmente en las anglosajonas donde la autonomía alcanza cotas muy superiores a la nuestra.

\subsection{Duración de los estudios y ciclos universitarios}

Nuestras enseñanzas han solido tener un carácter rígido. La estructura de las enseñanzas universitarias en España anteriores a la Ley de Educación de 1970 eran fundamentalmente de un ciclo de cinco o más años que concluía con el título de Licenciado, Ingeniero o Arquitecto.

Con la Ley de 1970 parece un ciclo intermedio que se corona con título de Graduado, Ingeniero o Arquitecto Técnico de tres años de duración que podría prolongarse hasta los cuatro años.

Estos ciclos se mantienen en la Ley de Reforma Universitaria aunque resulta sorprendente que el artículo 30 diga que "los estudios universitarios se estructurarán, como máximo en tres ciclos".

En lo que claramente se insiste es en la tendencia a rebajar la duración de los ciclos.

Se distingue entre enseñanzas sin título intermedio de aquéllas que lo tienen. La enseñanzas que designa el Documento como de "corta duración", podrían ser de dos o de tres años, y en las de "larga duración" se recomienda la estructura de cuatro años (dos + dos) "para no alargar innecesariamente los estudios con el consiguiente coste individual y social. También se contempla un primer ciclo que pudiera tener entre dos y tres años, y el segundo sólo de dos, excepto tres para Medicina y Odontología".

En las enseñanzas con título intermedio debería generalizarse el sistema de un primer ciclo de dos años y otro segundo de la misma duración, aunque también podría implantarse el de un primer ciclo de tres años y un segundo de dos años.

En "La Reforma" se detecta una tendencia a conservar la estructura actual con "un primer ciclo de tres años de duración (excepcionalmente de 
dos) conducente a la obtención del título de diplomado, arquitecto técnico o ingeniero técnico y un segundo ciclo, de dos años de duración conducente a la obtención del título de licenciado, arquitecto o ingeniero". Para estos últimos títulos se dan dos modelos: la enseñanza de dos ciclos sin titulación intermedia y la de dos ciclos con titulación intermedia. En este segundo caso la resistencia de las universidades suponemos que dejará las cosas como están ahora. La ley de Educación de 1970, también preveía esta situación y de hecho quedó sólo en el texto legal. Entre el "Documento" y la "Reforma" la tendencia a la reproducción temporal y a la implantación de los dos ciclos para todos los estudios ha ido cediendo posiciones y volviendo a las estructuras actuales.

En la "Reforma" se puntualiza que "las enseñanzas se ordenarán por ciclos y no por cursos académicos, de modo que los estudiantes podrán organizar sus estudios con amplia libertad, matriculándose de más o menos asignaturas, según sus disponibilidades para el estudio" No se trata de cursos anuales en fijas secuencias de materias al uso. El estudiante podrá ir superando la carga lectiva correspondiente a cada título, a un ritmo personal, si bien podrá establecerse el mínimo y el máximo de escolaridad obligatoria, pero de hecho "la duración efectiva de los estudios variará para cada estudiante".

Esta tendencia a la reducción de los años de estudios necesarios para alcanzar el título y esta diversificación temporal de acuerdo a los proyectos de los alumnos rompe esquemas tradicionales y da un nuevo acento a nuestros currículos.

El escalonamiento de los tres ciclos se plantea asimismo con una gran flexibilidad. El primer ciclo no es la vía única para acceder al segundo de determinada carrera. Se puede pasar del primero a más de un segundo ciclo con los complementos formativos (CF) que se establezcan.

Quizá el primer ciclo sea el más novedoso y el que implica una mayor elaboración por parte del Consejo de Universidades. En el pleno del Consejo del 14 de Octubre de 1985 se recomendó "potenciar y diversificar las enseñanzas del primer ciclo, incentivando su carácter terminal". Aunque se insiste en que tendrá entre dos y tres años de duración, sin embargo, en todos los esquemas propuestos en el Documento aparece el modelo de los tres años, lo cual resulta revelador.

Contando con nuestra tendencia a prolongar los estudios por razones exclusivamente académicas y escasamente profesionales, es de prever que este modelo trianual dominará en los nuevos currículas universitarios.

Aunque en la Ley General de 1970 se preveía la implantación generalizada de este primer ciclo, incluso para las tradicionales carreras largas, sin embargo, de hecho quedó limitado a las escuelas Universitarias, que lo impartían con carácter terminal, si bien mediante un curso de acceso podían 
los titulados pasar al segundo ciclo de Facultades y Escuelas Superiores. Este modelo se propone como uno más, pero ahora se pretende que incluso las enseñanzas de larga duración tengan un primer ciclo que conduce a título terminal, que tendrá su continuidad natural en el segundo ciclo y que equivaldría a la posesión del título de diplomado.

\subsection{Los planes de estudios}

Este es el punto decisivo del Documento en cuanto concierne al tema del curriculum, el que implica mayores novedades y el que posiblemente suscite mayores reservas.

\subsubsection{Directrices generales para los planes de estudios}

Estas directrices que serán motivo de un decreto del Gobierno a propuesta del Consejo de Universidades, serán de obligado cumplimiento por todas las Universidades que quieren otorgar los títulos oficiales de validez nacional.

Según estas directrices como mínimo existirá un 30\% y como máximo un $50 \%$, correspondiente a la carga de materias troncales. Todas las universidades deberán incluir estas materias que "podrán definirse como asignaturas o como conjunto de asignaturas".

Un 60\%, como máximo, serán determinados por la Universidad, de las cuales existirán dos bloques: uno de materias obligatorias para alcanzar determinado título y, otro de materias optativas entre las cuales podrá elegir el alumno.

Queda todavía un 10\% como mínimo que el alumno escogerá libremente de entre los cursos impartidos en la Universidad conducentes a títulos oficiales.

Además de estas normas generales existen otras directrices correspondientes a cada título. La Cominsión XV establecida por el Consejo de universidades, es la encargada de establecer las condiciones de los estudios que actualmente conducen a los títulos de profesor de Educación Básica y $\mathrm{Li}$ cenciado en Ciencias de la Educación.

En estas directrices, según el Documento, figurarán:

- La denominación de cada título

- Contenidos básicos de las enseñanzas

- El perfil profesional

- Aspectos metodológicos y pedagógicos

- El carácter cíclico de las enseñanzas y los requisitos para pasar de un ciclo a otro. 
- Se determinarán los requisitos de entrada o comienzo de estudios y los terminales, tales como tesina, proyecto de fin de carrera, examen o prueba general, o sencillamente haber aprobado todos los créditos requeridos.

- Periodo de escolaridad mínima a tiempo completo o a tiempo parcial para los estudiantes que trabajan.

- Ordenación secuencial estableciendo, en su caso, incompatibilidades de materias o créditos necesarios sean teóricos, prácticos o clínicos.

- Se especificarán las áreas de conocimiento a las que se adscriben las materias.

\subsubsection{Los créditos}

Frente a nuestro tradicional planteamiento de cursos anuales y en algunos casos semestrales, que imponían una rígida secuencia temporal que obligaba a cursar cinco materias anuales, se propone una flexible articulación de las enseñanzas distribuidas en créditos.

La propuesta del Consejo de Universidades es clara: "Conscientes de la conveniencia de que el sistema de créditos se generalice en la ordenación de las enseñanzas de nuestra Universidad, la Ponencia sugiere se defina este mecanismo de créditos en el nivel normativo correspondiente, a cuyo efecto propone:

- La definición del crédito como unidad que cuenta en términos del crédito $=10$ horas de carga lectiva. Los créditos harán referencia a su contenido teórico, práctico o, en su caso, clínico."

\subsubsection{Estudio y trabajo}

En España ha sido usual que el título universitario habilitase directamente para el ejercicio profesional, con el único requisito en algunos casos de la previa colegiación. Esta vinculación que no se da, o se da en menor escala en otros países entre lo académico y lo profesional, no ha impedido que nuestras enseñanzas, según el Documento que comentamos "se hayan caracterizado, en muchos casos, por un excesivo academicismo y un notable desfase entre las formaciones académicas recibidas y las posteriores exigencias profesionales que, precisamente, eran postuladas por el título. En otras ocasiones ha sucedido que profesiones socialmente demandadas ( $\mathrm{Di}$ seño, Terapia Ocupacional, etc) no han merecido una previa consideración académica articulada en estudios reglados."

En el documento se insiste en la vinculación de la teoría y la práctica y en que, cuando se formulen los créditos, aparezcan de una manera explícita ambas facetas. 
Quizá el aspecto más novedoso sea el punto IX.IV donde se habla "Posibilidad de alternancia entre estudio y trabajo (cursos "sandwich") valorable en el curriculum".

De los modelos que propone el Documento para los planes de estudios se ha tenido buen cuidado y con un carácter excepcional, de visualizar en forma de esquema las propuestas, para que tuvieran un carácter más intuitivo y aún persuasivo.

Transcribimos los tres modelos que presenta el documento que analizamos.

En el primero de los que exponemos se puede comprobar que se equilibran los contenidos académicos $(\mathrm{A})$ y los profesionales $(\mathrm{P})$, aunque estos últimos van aumentando al paso de los años. A pesar del gráfico se insiste en que la carga lectiva tendrá una duración equivalente a dos o tres años. El título conduce directamente al segundo ciclo.

\section{GRAFICO I}

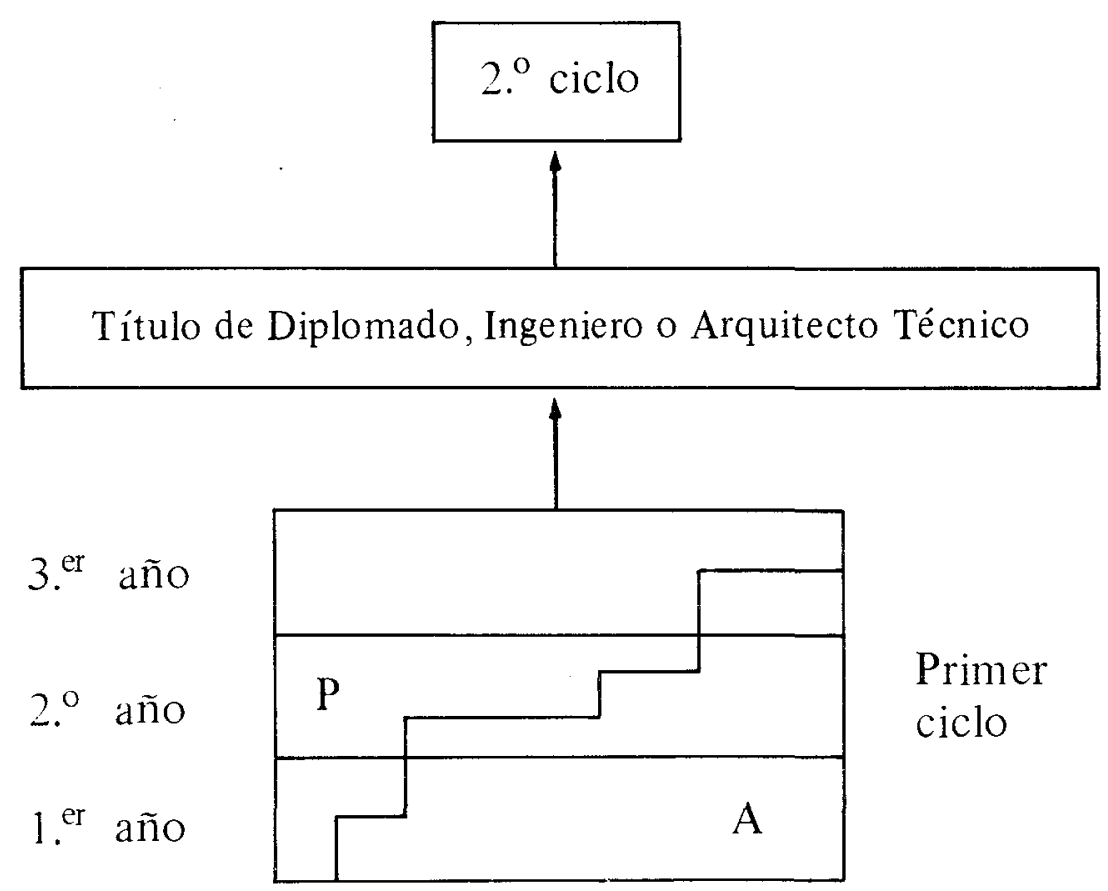

El modelo siguiente, manteniendo las mismas características temporales y de equilibrio entre los contenidos profesionales y los académicos, por no poder compatibilizar la formación profesional con la académica necesaria para el segundo ciclo, requiere incluir créditos académicos (CA) de naturaleza científico-técnica que permita acceder al siguiente ciclo. Estos complementos o créditos académicos pueden recibirse antes o después de alcanzar el título. 


\section{GRAFICO II}

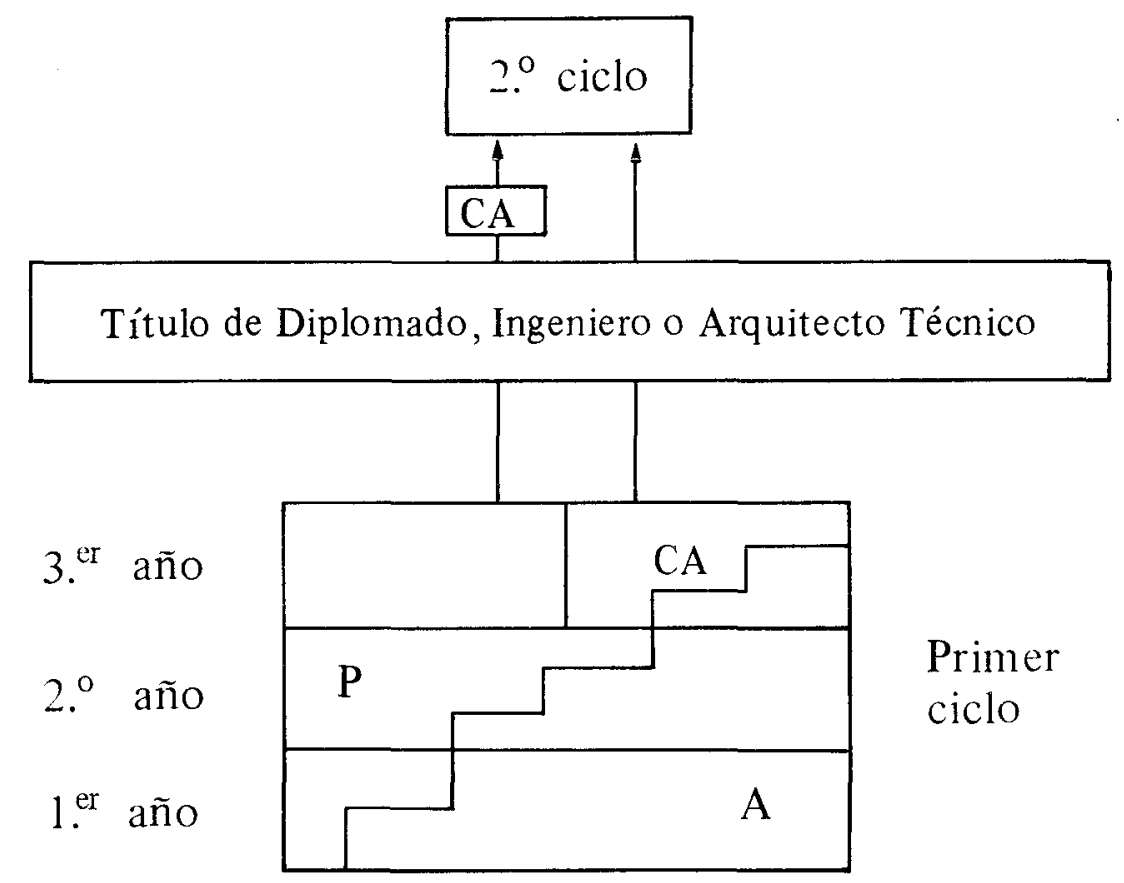

El tercer esquema presenta dos tipos de diplomatura, una más académica y generalista, que lleva directamente al segundo ciclo, y otra más profesional que requiere un complemento de formación académica.

\section{GRAFICO III}

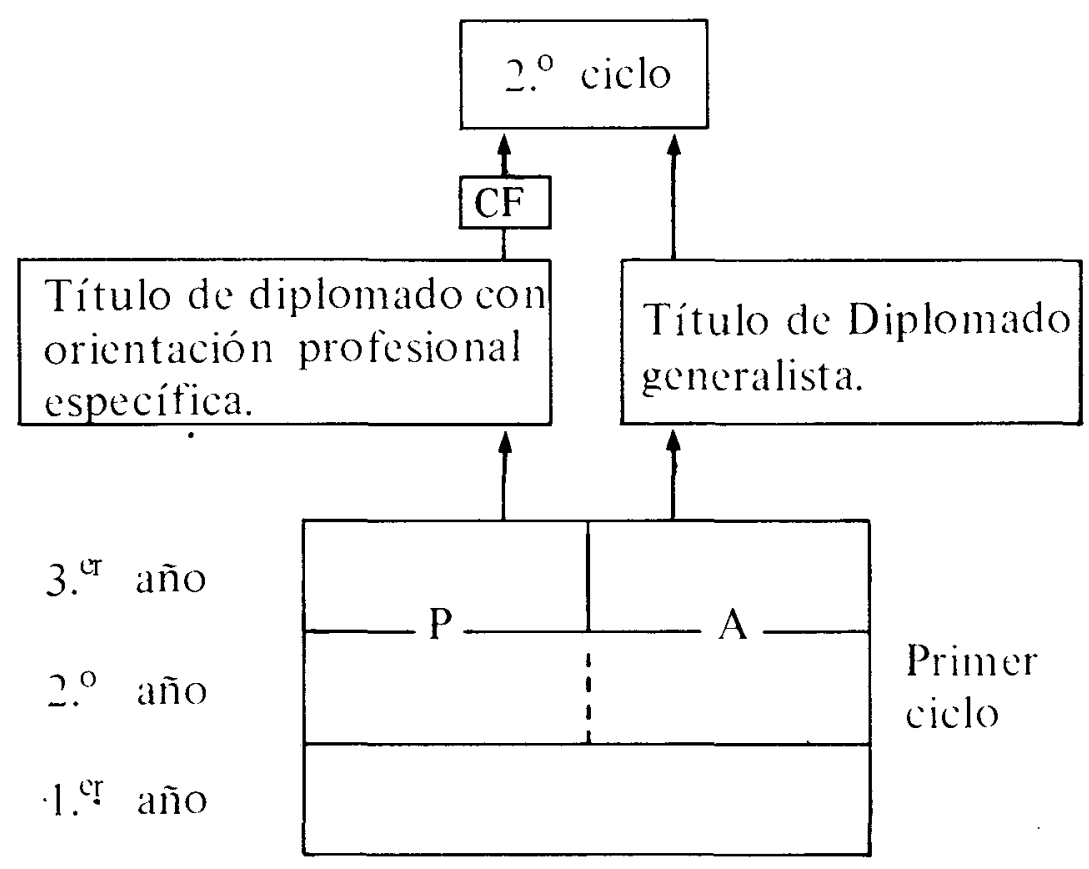


En cualquier caso, la preocupación por insistir en la dimensión profesional queda patente.

Uno de los aspectos más novedosos es el de sugerir que en las directrices que establezcan para los planes de estudios de específicas titulaciones, debe aparecer junto al contenido básico de las enseñanzas, el perfil profesional. Posiblemente este será uno de los puntos más conflictivos y de difícil cumplimiento dada la rápida evolución de la población activa y de los perfiles profesionales. Además de la incierta y móvil frontera entre la formación en el Centro docente y la preparación inmediata para un concreto puesto de trabajo.

\subsubsection{Actualización de los planes de estudio}

Es grave problema la actualización de las enseñanzas, a la que fuerza un crecimiento exponencial de los conocimientos y una desbordante aportación de nuevos métodos, técnicas, datos y teorías.

Para ello habrá que prever "formulas de evaluación intrauniversitaria en orden a la actualización del plan con carácter periódico". Por ello el documento se cierra con la indicación de que" Deberán establecerse, bien en directrices generales básicas o en directrices propias de título, los correspondientes mecanismos de extinción progresiva de los planes, sin perjuicio de que pueda instaurarse al tiempo un mecanismo de adaptación a los nuevos planes."

\section{LA REFORMA DE LAS ENSEÑANZAS UNIVERSITARIAS A LA LUZ DE LAS TENDENCIAS INTERNACIONALES}

Cada una de las propuestas que aparecen en el programa para la reforma de las enseñanzas universitarias, merece una consideración específica. La primera es la de convertirlo en una cuestión, encerrarla entre interrogantes y preguntar por sus fundamentos, validez y consecuencias.

El documento se inscribe en una línea de política educativa que dará lugar a las planificaciones y programaciones correspondientes. Y la primera condición para el éxito de toda planificación es la de un análisis cuidadoso de la realidad, para lo cual se requieren numerosas investigaciones, que poseemos en muy escasa medida y que no tenemos información de que estas pocas se hayan tenido muy en cuenta. Se trata de propuestas de afán renovador, impulsadas por razones políticas y de obligado desarrollo normativo de la ley, más que por los imperativos de una rigurosa planificación y por exigencias de la innovación educativa, tema éste que goza de una dila- 
tada bibliografía a partir de los años 50 y que ha sido motivo de recomendaciones en el ámbito internacional. La obra "Innovación y problemas de la educación" (Unesco, 1980) pudiera ser indicativa de esta tendencia, la cual no creemos que haya sido tenida en cuenta.

Nos vamos a circunscribir a algunos de los temas capitales formulados en el documento y aportar la información internacional que nos permita de algún modo contrastar su validez. Dada la multiplicidad de factores que entran en juego en el sistema educativo, inserto en el siștema social, una de las vías de evaluación más eficaces, es la de comprobar en el ámbito internacional las tendencias emergentes, como fruto y depuración de unas experiencias dilatadas en el tiempo y realizadas en las más varias condiciones sociopolíticas, económicas y culturales.

\subsection{La duración de los estudios}

Un análisis comparado de la duración de los estudios en diversos países, especialmente en los europeos y anglosajones revela que en general nuestras enseñanzas tienen una duración excesiva.

Hemos seleccionado algunos grandes países, que por su proximidad geográfica o por su indudable impacto en el el mundo contemporáneo, son reveladores de las tendencias en alza. Los datos han sido tomados de la obra de la UNESCO: "Les études superieures. Présentation comparative des régimes d'enseignement et des diplômes".

GRAFICO IV

T I T U L O S

\begin{tabular}{|c|c|c|c|c|c|c|c|c|}
\hline PAINOS & 1 & 3 & 4 & 5 & 6 & 7 & 8 & \\
\hline ESPAÑA & & $\begin{array}{l}\text { Graduado } \\
\text { Ingeniero } \\
\text { Técnico }\end{array}$ & & Licenciado & Ingeniero & Doctor & & \\
\hline USA & $\begin{array}{l}\text { Associate } \\
\text { Certificate }\end{array}$ & & Bachelor & & Master & & Doctor & \\
\hline $\begin{array}{l}\text { ALEMANIA } \\
\text { FEDERAL }\end{array}$ & & & Diplom & & & Doctor & & \\
\hline FRANCIA & $\begin{array}{l}\text { Diplôme } \\
\text { Universit } \\
1 .^{{ }^{2} \mathrm{~T}} \text { cycle }\end{array}$ & $\begin{array}{l}\text { Licence } \\
\text { Diplôme }\end{array}$ & Master & Diplôme & Doctorat & & $\begin{array}{l}\text { Doctorat } \\
\text { d'Etat }\end{array}$ & d'Etat \\
\hline $\begin{array}{l}\text { REINO } \\
\text { UNIDO }\end{array}$ & & Bachelor & Master & Doctor & & & & \\
\hline ITALIA & $\begin{array}{l}\text { Diploma } \\
\text { profoss. }\end{array}$ & $\begin{array}{l}\text { Diploma } \\
\text { profoss. }\end{array}$ & Laurea & & $\begin{array}{l}\text { Diploma } \\
\text { specializz }\end{array}$ & & & \\
\hline
\end{tabular}


Contando con las inevitables simplificaciones a que fuerzan estas visiones de conjunto, se pueden extraer algunas conclusiones. Efectivamente nuestros estudios universitarios se prolongan en exceso. Sin embargo la limitación a tres ciclos "como máximo" no responde a la diversificación creciente de los títulos universitarios, de acuerdo a una demanda múltiple. De hecho en todas las reformas se va imponiendo la proliferación de titulaciones cortas.

En España todas las enseñanzas postsecundarias son universitarias desde la Ley de Educación de 1970. Pero esta situación se da sólo en 1/3 de los países. Los que poseen enseñanzas terciarias no universitarias tienen una gran variedad de títulos, respondiendo a una demanda cambiante y en expansión incesante. Entre nosotros es pues más urgente diversificar los títulos y sus exigencias temporales, que no puede responder a rígidos esquemas académicos previos, sino a los imperativos de los perfiles profesionales.

\subsection{Los créditos}

Los créditos tienen una clara raíz anglosajona y aunque es un sistema indudale de flexibilizar los currículos, no es el único. En algunos países se habla de unidades modulares y capitalizables. Los créditos en Norteamérica presentan una temporalización diferente a los que se pretende configurar en España. Un crédito son 15 horas de clases lectivas o de lecciones magistrales que implica unas 30 horas de estudio para asimilarlas, lo que suma unas 45 horas de trabajo para el alumno. Cuando se trata de actividades prácticas tales como laboratorio hay que cumplir 45 horas y cuando se refiere a seminarios, 30 horas de éstos suponen 15 de trabajo por parte del alumno.

A título de ejemplo transcribimos el sentido que tiene en una universidad típica en U.S.A. la Wayne State University: "Un crédito (crédito hora) se define como una hora de clase semanal o su equivalente reconocido, que requieren un mínimo de dos horas semanales de preparación, a lo largo de un semestre. En otras modalidades de instrucción el crédito deberá ser lo más coherente posible con la anterior definición.

Laboratorio: Tres horas de laboratorio se consideran equivalentes a una hora de clase".

En España el diseño corresponde a diez horas teóricas o prácticas. Este hecho, al parecer intranscendente, puede crear problemas de cara al futuro. A la hora de convalidar títulos, de establecer equivalencias y reconocimientos de estudios, el elemento temporal resulta decisivo y habrá que ver las implicaciones de una legislación que, al menos por el momento, puede establecer una temporalización singular de los créditos, más sorprendente cuando la Ley de Reforma Uniersitaria ha contemplado explícitamente "la movilidad de titulados españoles y extranjeros". 
Por buscar algunos de nuestros precedentes habría que recordar el Certificado de Aptitud Pedagógica (CAP), expedido por los ICES, donde se sumaban los cursos - con un mínimo de 20 horas cada uno- dentro de unos bloques fundamentales, pero con una gran flexibilidad de acuerdo a las innovaciones educativas y a las demandas de los usuarios.

Lo que parece evidente en la tendencia moderna, es que los estudios pueden estructurarse de acuerdo con algunas líneas fundamentales, y en donde la opcionalidad del sujeto tiene un espacio creciente de acción. Entre nosotros donde la rigidez académica ha llegado a extremos hace tiempo superados en casi todos los países, esta flexibilización es la única salida posible para los planes de estudio elaborados por la universidades, que van a presentar una variabilidad mucho más acentuada de la registrada hasta aquí.

\subsection{Formación y trabajo}

Para subrayar la importancia del tema bastaría recordar que la Conferencia Internacional de Educación de Ginebra, ha formulado dos Recomendaciones, lo que revela su interés, la primera en 1973, sobre "los vínculos entre la educación, la formación y el empleo, especialmente en lo que respecta a la enseñanza secundaria, sus objetivos, estructura y contenido" y en 1981 sobre "Interacción entre la educación y el trabajo productivo".

El IX Congreso Internacional de la Asociación Mundial de Ciencias de la Educación que tuvo lugar en Madrid del 8 al 12 de julio de 1985, el tema fue "La educación y el trabajo en la sociedad moderna".

Sería difícil establecer una relación exhaustiva de los Congresos, Seminarios, Mesas redondas, artículos, libros y números monográficos de las revistas, dedicados a la vinculación entre estudio y trabajo.

Ante la inevitable selección y puesto que nos situamos en una perspectiva internacional, parece pertinente recurrir a la documentación de la UNESCO y de una manera específica a la obra "Worl-wide inventary of nontraditional postsecundary educational institutions" (UNESCO, 1980, segunda revisión actualizada, 1984). La importancia de la obra puede constatarse porque fue aprobada explícitamente para el programa y presupuestos de la UNESCO en el bienio 77-78, y para la segunda edición en el del trienio 81-83. La necesidad de esta obra surgió porque las nuevas exigencias sociales imponen la remodelación de la educación superior contemplada como un factor capital del desarrollo de los países, directa o indirectamente, a través de la investigación y el trabajo de sus titulados. Hay que atender a nuevas clientelas y para responder a nuevos objetivos, aparecen contenidos, métodos y sistemas administrativos que hagan a la universidad más eficiente en el plano interno y en el externo. 
Las fórmulas propuestas para esta vinculación de la universidad y el trabajo son muchas, una de ellas demanda que la universidad se convierta en un elemento productivo y que sus alumnos, los dedicados a tiempo completo a la universidad, al realizar sus prácticas elaboren productos vendibles en el mercado. Esta concepción frecuente en los países marxistas quizá sea una situación límite, circundada en problemas, pero en cualquier caso merece ser estudiada. El Seminario sobre relación entre educación, investigación y producción en la enseñanza superior que tuvo lugar en Sofía en 1980 bajo el patrocinio de la UNESCO, es indicador en esta línea.

Analizando el "World-Wide Inventory" puede comprobarse que en prácticamente todos los países, cuando se habla de instituciones innovadoras o no tradicionales, se insiste en la vinculación de trabajo y estudio. La concesión de créditos al ejercicio profesional relacionado con los planes de estudios, la organización de las enseñanzas a tiempo parcial para poder simultanear estudio y trabajo son dos modalidades de uso frecuente que imponen una nueva configuración de los currículos. Las prácticas escolares realizadas no sólo en los laboratorios de las universidades, sino en las empresas y todo tipo de instituciones donde previsiblemente actuarán los titulados universitarios, es una técnica en expansión, incluso en países donde el rigor académico parecía poco compatible con las actividades laborales. Médicos, abogados y pedagogos sociales invierten una parte considerable de su tiempo y realizan una parte de su trabajo, en las propias instituciones donde actuarán en su vida profesional.

Los criterios que ha empleado la UNESCO, en el citado documento, al seleccionar las instituciones no tradicionales o innovadoras y que en su mayor parte han aprendido a partir de los años sesenta, son los siguientes:

- Centros que están abiertos a nuevos sectores de la población, tradicionalmente desatendidos por las universidades, las que se han circunscrito a jóvenes de 18-25 años, sin empleo y que han completado la educación secundaria.

- Flexibilidad en los tiempos de estudio lo que permite compatibilizarlo en empleo a tiempo parcial.

- Estrecho contacto con industrias, servicios públicos y otras instituciones que emplean personal con educación superior.

- Establecimientos dedicados a la actividad profesional y que conceden diplomas o certificados.

- Centros que ofrecen variadas posibilidades para actualizar a los profesionales y están empeñados en actividades industriales $u$ otras ocupaciones.

- Hacer un uso extensivo de la nueva tecnología educativas tales como radio, televisión, teléfono, computadores, etc para alcanzar nuevas poblaciones, modificando los tradicionales modelos organizativos. 
Si tenemos en cuenta estos criterios - que han permitido seleccionar miles de instituciones- nuestras universidades tienen poco de innovadoras. Las implicaciones de una estrecha relación trabajo-estudio, en parte, se han tenido en cuenta en las directrices del Consejo de Universidades, pero nuestra tradición centralista -aunque lo sea también a nivel autonómico- y los rígidos marcos legales habrán de ser utilizados con enorme flexibilidad para que la renovación de nuestros currículos vaya más allá de las declaraciones políticas y de los textos legales.

La vinculación de trabajo y estudio obliga a flexibilizar las plantillas del profesorado, incluyendo las figuras relevantes en el campo social que puedan aportar sus creaciones al ámbito universitario.

La propia investigación adquiere de manera creciente un acento más vinculado con las necesidades sociales. La industria química alemana o la aeroespacial norteamericana, nutren sus espectaculares avances de la investigación realizada en convenios con la universidad.

La recomendación de que al formular los currículos universitarios se tenga en cuenta el perfil de los profesionales en ejercicio y más aún las exigencias más o menos previsibles de cara al futuro laboral, tiene un impacto creciente en otros países y está prácticamente desconocida entre nosotros. En cualquier caso esta es una de las vias más prometedoras para la renovación de los currículos universitarios.

\subsection{La Educación permanente y los currículos innovadores}

Sorprende que en la renovación de nuestros currículos universitarios no aparezca ninguna alusión explícita a la educación permanente. Esto puede tener graves consecuencias para el diseño de nuestra universidad. En el citado "Inventario mundial de instituciones postsecundarias educativas no tradicionales", en realidad el principio impulsor de todas las renovaciones es el de la educación permanente, así aparece explícitamente formulado y su proyección se constata a lo largo de las páginas densas de esta obra.

Ya en 1971 la UNESCO lanzó un programa a largo plazo para impulsar las nuevas tendencias en la educación superior en la línea de la educación permanente con el propósito fundamental de renovar los sistemas educativos.

En la resolución 1371 adoptada por al Conferencia General en su 17 sesión, autorizaba al Director General a estudiar los problemas de la educación superior, prestando especial atención a la educación permanente renovando los métodos y contenidos.

Se han centrado los esfuerzos en las siguientes áreas: nuevas funciones de las instituciones de enseñanza superior en el contexto de la educación 
permanente; nuevas estructuras, atención a las personas empleadas y a los servicios comunitarios.

Desde el comienzo de los años 70 se ha prestado atención a las proyecciones prácticas como al concepto de educación permanente. En este sentido y para alcanzar nuevas clientelas, respondiendo a las crecientes demandas, se han estudiado los nuevos métodos y recursos, en el programa de investigación UNESCO Asociación Internacional de Universidades, que cuajó en la obra: "Teaching and learning -an introduction to new methods and resources in higher education" (1970).

En 1974 tuvo lugar en Moscú un simposium sobre "El papel de la educación superior en la educación permanente".

Un comité de expertos en 1977 examinó las modalidades en las cuales la educación permanente ha formado parte de la vida universitaria. El profesor G. Williams de la Universidad de Lancaster publicaba la obra "Towards Long-Life Education: A New Role for Higher Education Institutions (UNESCO, 1977).

En 1976 tenía lugar en Londres una reunión de expertos sobre la educación postsecundaria para personas en pleno empleo donde se estudiaron casos en varios países occidentales y orientales. $\mathrm{Al}$ año siguiente un Comité de expertos estudiaba las vías para que se pudiese instaurar la educación permanente como una actitud normal de la universidad.

En la 19 sesión de la Conferencia General, se adoptó una resolución para diseminar la información en retorno a este tópico. El secretariado de la UNESCO también recogió información a través de las comisiones regionales y de más de trescientas sesenta instituciones para que completaran el cuestionario que dio lugar a este inventario. En 1979-80 se realizaron estudios en profundidad y comparativos sobre este campo y en 1983 se terminó su elaboración. La preocupación por el tema sigue viva en el ámbito internacional.

De hecho nuestro sistema educativo parece ignorar el principio de la educación básica y éstas con una proyección muy limitada.

Nuestra universidad parece rechazar los alumnos que no puedan dedicarse a tiempo completo a los estudios. No se cuida de los titulados que terminaron años atrás y que necesitan la actualización profesional sin la cual no cabe un desarrollo socioeconomico y cultural.

Sólo la Universidad a Distancia está diseñada para atender a la población permanente de los profesionales. Lo cual es habitual en todas las universidades a distancia e incluso en muchas universidades extranjeras.

En los Estados Unidos hay aproximadamente 12 millones de alumnos dedicados exclusivamente a la Universidad hasta obtener la titulación, pero los programas destinados a educación permanente, especialmente del profesorado, afectan a 17 millones. 
Pero lo importante desde el punto de vista del curriculum, es que si se cuenta con la educación permanente, desaparece la obsesión por aumentar ilimitadamente los programas, por recargar los contenidos teóricos que tendrán menos oportunidades de adquirir en la vida profesional y por convertir los estudios universitarios en una información enciclopédica que pueda resolver todas las situaciones futuras. El crecimiento constante de las informaciones y las nuevas investigaciones, dejan obsoleto pronto gran parte de lo enseñado en las aulas. Mas no se trata sólo de una cuestión de cantidad y de selección, ni de distribuir la formación a lo largo de toda la vida, sino del sentido mismo de las enseñanzas universitarias. Estas deben destacar los conceptos y teorías, prácticas y métodos que tengan una función polivalente y que permitan la inserción en variadas actividades, muchas de ellas imprevisibles.

\section{CONCLUSIONES}

A pesar de las limitaciones apuntadas: la motivación político-legal, la carencia de estudios e investigaciones previas y la poca referencia a la auténtica línea renovadora de los currículos desde el ángulo de la educación permanente; estimamos que el intento de flexibilizar los currículos a través de los créditos, la reducción del tiempo dedicado a los estudios, la vinculación con el mundo laboral a través de la diversificación de los títulos profesionales, del aumento de las prácticas - que para serlo deben tener un acento tecnológico, es decir, aplicación de conocimientos científicos- y la posibilidad de compatibilizar estudio y trabajo y más aún de conceder a éste valor académico, son líneas prometedoras en las que merece empeñarse para superar patentes deficiencias de nuestros currículos universitarios. 


\section{BIBLIOGRAFIA}

ASSOCIATION MONDIALE DES SCIENCES DE L'EDUCATION (1986). L'education et le travail dans la société moderne. Madrid. Anaya.

BLAT GIMENO, J. y MARIN IBAÑEZ. R. (1980) La formación del profesorado de educa. ción primaria y secundaria. Estudio comparativo internacional. Barcelona/ París.Teide/ UNESCO.

CONSEJO DE EUROPA (1970). Education permanente. Estrasburgo (1973) Educatión permanente. Principes de base. Estrasburgo.

HAVELOCK, R.G. y HUBERMAN, A.M. (1986) Innovación y problemas de la educación. París. UNESCO

INTERNATIONAL ASSOCIATION OF UNIVERSITIES. bulletin (Trimestral) París.

MARIN IBAÑEZ R. y otros (1984) Sistematización e innovación educativas. Madrid. UNED. MINISTERIO DE EDUCACION Y CIENCIA. (Madrid)

- (1983) Ley de Reforma Universitaria

- (1986) Programa de actuación para la reforma de las enseñanzas universitarias. Consejo de Universidades.

- (1987) La Reforma de las Enseñanzas Universitarias. Consejo de Universidades

ORGANISATION DE COOPERATION ET DE DEVELOPPEMENT ECONOMI-

QUES. (Centre pour la Recherche et L'Innovation dans l'Enseigment). Revue Internationale de Gestion des Établissements d'Enseignement Supèrieur. París.

UNESCO. París. (1984) Les études supérieures. Présentation comparative des régimes d'enseignement et des diplômes.

- (1979) Conferencia Internacional de Educación. Recomendaciones 1934-77

- (1981) Convenciones, Recomendaciones y Declaraciones de la UNESCO. Comisión Nacional Española de Cooperación con la UNESCO. Madrid.

- (1984) World-Wide Inventory of Non-Traditional Post-Secondary Educacional Institutions. UNESPO-CEPES. Enseignement superieur en Europe (Revista trimestral del Centro Europeo para la enseñanza superior). Bucarest. Rumanía.

WAYNE STATE UNIVERSITY. Bulletín 1985-86. Detroit (USA) 